\title{
I SOMMERSI E I SALVATI. PRIMA EDIZIONE SCOLASTICA COMMENTATA DELL'OPERA DI PRIMO LEVI
}

di Martina Mengoni and Roberta Mori

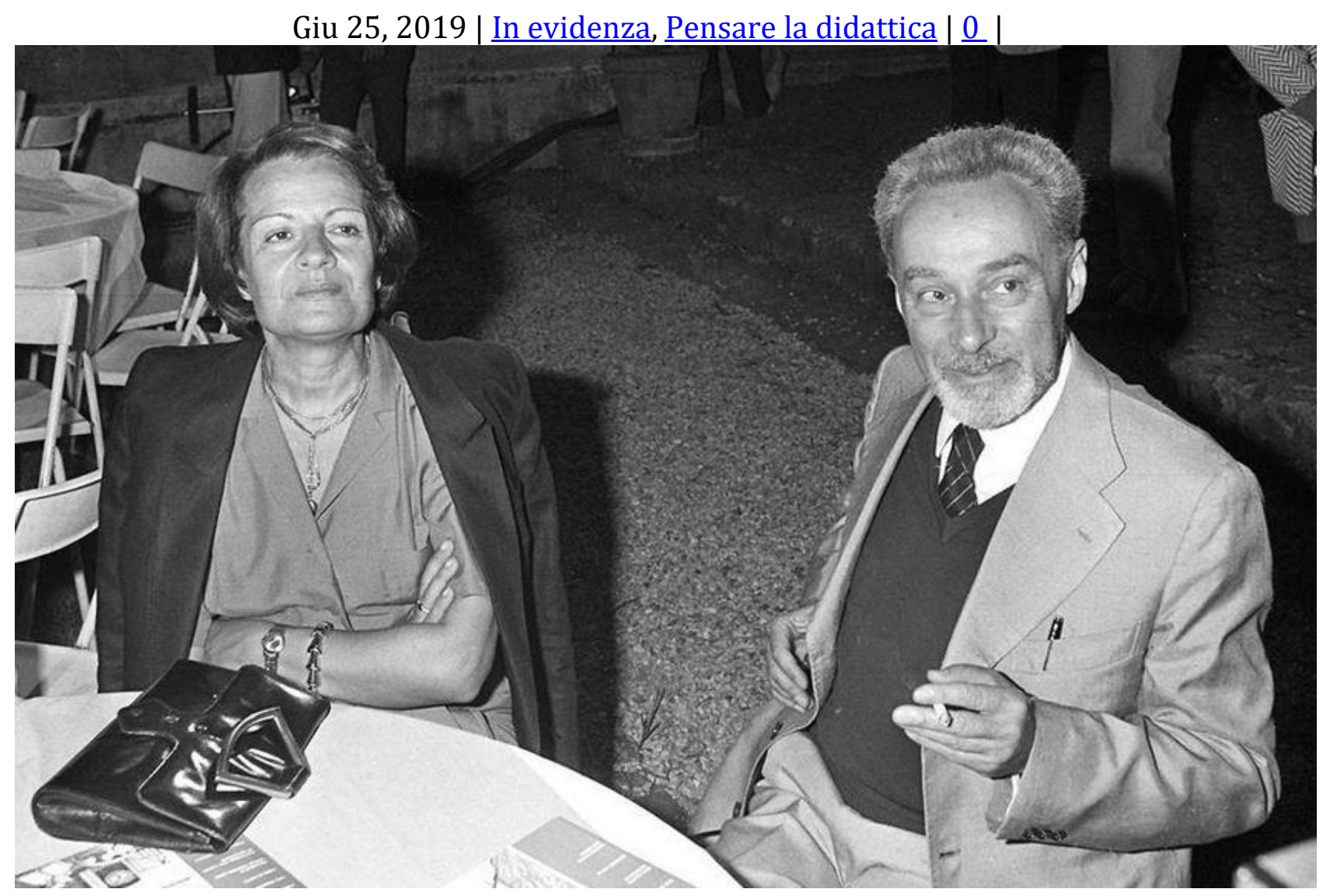

rimo Levi con la scrittrice Francesca Sanvitale in occasione di un'edizione del Premio Streg.

Foto dii sconosciuto - http://www.corriere.it/foto-gallery/cronache/16 luglio 25/31-luglio1919-nasceva-primo-levi-7734a32c-4bf1-11e6-9b53-09d4e26665fb.shtml, Pubblico dominio $\underline{\text { Collegamento }}$

\section{ABSTRACT}

Nel mese di marzo è stata pubblicata per la prima volta un'edizione scolastica commentata de I sommersi e i salvati di Primo Levi che comprende un volume per gli studenti e un fascicolo per gli insegnanti: nel primo si trova il testo di Levi preceduto da un'introduzione rivolta ai ragazzi e accompagnato da un apparato di note ed esercizi sul testo, il secondo contiene invece una serie di proposte didattiche aggiuntive che i docenti possono sviluppare in classe. Nell'articolo le due curatrici chiariscono i presupposti metodologici alla base delle scelte compiute nella pubblicazione e descrivono, attraverso una selezione di esempi, i contenuti principali delle proposte didattiche e il loro possibile impiego all'interno di una didattica innovativa delle discipline storico-letterarie. 
Nel mese di marzo è stata pubblicata, per i tipi di Einaudi Scuola, un'edizione scolastica commentata de $I$ sommersi e i salvati di Primo Levi che comprende il testo dell'opera preceduto da un'introduzione e accompagnato da un apparato di note ed esercizi sul testo, corredata da un fascicolo con proposte didattiche aggiuntive destinato ai docenti.

L'edizione scolastica è il frutto di un percorso di ricerca letteraria e didattica condotto dal Centro Internazionale di Studi Primo Levi di Torino. La progettazione e la realizzazione delle note al testo, degli apparati didattici del volume e del fascicolo per gli insegnanti hanno fornito l'occasione per compiere una riflessione a tutto campo sulle possibili modalità di trasmissione dei contenuti dell'opera, uno dei pochi testi di Primo Levi mai pubblicato in edizione scolastica.

\section{ANALISI LETTERARIA E MEDIAZIONE DIDATTICA}

Obiettivo principale del lavoro di editing è stato la ricerca di risposte didatticamente soddisfacenti alle molte esigenze poste da una materia delicata e complessa come quella affrontata da Levi: come aiutare gli studenti a comprendere tutti i riferimenti presenti nel testo senza appesantire la pagina con note troppo estese? Come favorire una lettura attiva e partecipe? Come stimolare la riflessione sui concetti-chiave? Infine, e soprattutto: come far capire ai ragazzi che I sommersi e i salvati non è un testo "imbalsamato", ma è capace di interrogare senza sosta il lettore, mettere in scena davanti ai suoi occhi contraddizioni insolubili, scandagliare con lucidità gli anfratti più dolorosi della storia del Novecento senza consegnare verità apodittiche?

Nel concepire la fisionomia generale del volume e il commento al testo, preziosi suggerimenti indiretti sono venuti dalle scelte compiute dall'autore in occasione dell'edizione scolastica del 1973 di Se questo è un uomo[1], curata dallo stesso Primo Levi e accolta nella collana einaudiana "Letture per la scuola media". In quel frangente, lo scrittore aveva messo a punto, oltre alle note, una serie di strumenti allo scopo di rendere l'opera pienamente accessibile al pubblico degli studenti delle scuole medie: una Prefazione ai giovani; due carte geografiche che mostravano rispettivamente la disposizione dei campi di concentramento nei territori occupati dalla Germania e l'insieme dei sottocampi presenti nella regione di Auschwitz; una bibliografia essenziale nella quale erano consigliate opere di carattere generale sul nazionalsocialismo e sulla persecuzione degli ebrei, insieme a documenti e testimonianze. In un'Appendice-aggiunta all'edizione scolastica nel 1976-Levi aveva raccolto le domande più frequenti che gli venivano rivolte dagli studenti durante gli incontri nelle scuole, formulando per ciascuna risposte che ancora oggi costituiscono una indicazione di metodo per quanti vogliano accostarsi alla didattica della Shoah. 


\section{L'ESEMPIO DI PRIMO LEVI: LE NOTE}

Le note curate da Levi per l'edizione scolastica di Se questo è un uomo possono essere raggruppate in cinque diverse tipologie: note di traduzione delle espressioni in lingue straniere; note di spiegazione di termini tecnici desunti da lessici specialistici (medico, militare, scientifico, letterario, filosofico etc.) e dal gergo del campo; note storiche e geografiche; note che illustrano il funzionamento del Lager; note di auto-commento delle scelte lessicali e dei riferimenti intertestuali. Il programma di storia delle scuole medie degli anni Settanta si fermava alla prima guerra mondiale; l'apparato di note, la prefazione, la bibliografia dovevano quindi fornire ai giovani lettori sintetiche quanto indispensabili informazioni sulla geografia e sulla storia europee tra il 1918 e il 1945 aiutandoli nel contempo a comprendere le condizioni di vita all'interno di un Lager nazista.

\section{L'EDIZIONE SCOLASTICA DE I SOMMERSI E I SALVATI}

\section{LE NOTE}

Possono essere sussunte sotto sei diverse tipologie, alcune delle quali ricalcano quelle dell'edizione scolastica di Se questo è un uomo:

1. note di traduzione dei termini stranieri;

2. note di spiegazione di termini desunti da linguaggi specialistici e di vocaboli di derivazione colta e letteraria, oggi di uso raro (ad esempio "scherani", "turpi", "mendaci", etc.);

3. note storiche che coprono un arco cronologico che si estende dalla Prima Guerra Mondiale fino agli anni Ottanta del Novecento;

4. note storico-biografiche che ricostruiscono nei tratti salienti la biografia dei tanti personaggi storici citati;

5. note di spiegazione delle citazioni esplicite riconducibili alla cultura classica, ai testi biblici e alle fonti letterarie;

6. note che chiariscono i diversi riferimenti culturali (libri, film, opere d'arte) cui l'autore ricorre per supportare alcuni passaggi argomentativi e per mettere in luce ulteriori sfaccettature del discorso.

Non sono state aggiunte al testo note di tipo critico-interpretativo per non sovrapporre la voce delle curatrici alla voce dello scrittore, e per non limitare le capacità interpretative degli studenti incanalandole in un'unica direzione.

\section{L'INTRODUZIONE}

Intende offrire agli studenti un resoconto accessibile delle ultime ricerche sulla genesi, la struttura, gli stili e l'impianto retorico de I sommersi e i salvati. L'ultimo libro di Levi è innanzitutto ricondotto alla sua prima gestazione, negli anni Sessanta, quando l'autore intratteneva uno rapporto vivo e diretto con i suoi lettori 
tedeschi. Al contempo, il libro non avrebbe visto la luce senza il dialogo ininterrotto con gli studenti delle scuole medie inferiori e superiori: "Sono stato in più di centotrenta scuole" scrive Levi nel 1979. Gli studenti sono dunque il destinatario ideale de I sommersi e i salvati; l'ultimo e forse il più importante lascito analitico di Primo Levi è per loro, ed è questa la chiave per comprendere le scelte retoriche e argomentative del libro: un testo che fonde l'impianto saggistico con l'andamento narrativo, ricco di "figure" memorabili e insieme inesauribili, che rilanciano gli interrogativi filosofici, morali e storici posti dall'autore; un libro dal carattere socratico, concepito come un tentativo di ripristinare la complessità di una vicenda storica, sociale, culturale: "una segnaletica di problemi", come lo ha definito la storica Anna Bravo.

\section{LA BIOGRAFIA E L'APPENDICE DI APPROFONDIMENTO}

Abbiamo inoltre assunto l'idea, già presente nella curatela di Levi, che per aiutare il pubblico scolastico a orientarsi nella storia dello sterminio non sia sufficiente annotare tutti i termini che richiedono un supplemento di spiegazione, ma siano indispensabili supporti specifici: per questa ragione, oltre all'introduzione e all'apparato didattico, il volume comprende una sintetica biografia di Levi ed è completato da un'appendice di approfondimento sui campi di concentramento e di sterminio. Nell'appendice è stata inserita una delle due carte geografiche comparse nell'edizione scolastica del 1973 di Se questo è un uomo, nella quale appaiono evidenziati i Lager citati ne I sommersi e i salvati

\section{GLI ESERCIZI SUL TESTO}

Agli esercizi presenti nel volume è affidata la funzione interpretativo-critica, non assolta da specifiche note al testo. Suddivisi per capitoli, sono pensati per mettere a fuoco i nodi fondamentali dell'argomentazione di ciascun tema, per proporre possibilità interpretative grazie alla giustapposizione con altri testi della tradizione letteraria e filosofica e per stimolare su di essi una riflessione attiva sotto forma di analisi lessicale e stilistica, di rielaborazione dei contenuti, di confronto intertestuale.

\section{L'ANALISI LINGUISTICA}

Gli esercizi di analisi testuale mirano ad ampliare il bagaglio linguistico degli studenti attraverso la riflessione sulle scelte dell'autore e l'approfondimento dei lessici specialistici; sul versante stilistico sono proposte soprattutto analisi di figure retoriche notevoli (similitudini, metafore, ossimori etc.) grazie alle quali è possibile pervenire a una comprensione piena delle stratificazioni semantiche presenti nel testo. Tra gli esercizi linguistici ne segnaliamo uno relativo al capitolo Comunicare, che promuove una riflessione sul 
fenomeno della "violenza fatta al linguaggio" nella Germania hitleriana e in particolare sul Lagerjargon, il gergo degradato parlato nei campi al quale il filologo ebreo Victor Klemperer dedicò pagine imprescindibili nel suo studio sulla lingua del Terzo Reich intitolato LTI (Lingua Tertii Imperi)[2]. Si richiede agli studenti di fare un piccolo "esperimento Klemperer" usando come libro-serbatoio I sommersi e i salvati e di costruire un glossario dei termini del Lagerjargon che potrà poi essere ampliato prendendo in esame anche altri testi, ad esempio Arcipelago Gulag[3]o lo stesso libro di Klemperer.

\section{LA RICERCA STORICA}

Alcuni esercizi comportano invece da parte degli studenti lo svolgimento di una vera e propria attività di ricerca storica. È questo il caso di un esercizio del capitolo La memoria dell'offesa in cui si propone di stilare una lista delle testimonianze scritte dai carnefici nazisti, distinguendo fra quelle pubblicate prima del 1986 e quelle pubblicate successivamente, e di due esercizi riferiti rispettivamente ai capitoli Intellettuale ad Auschwitz e Lettere di tedeschi. Il primo suggerisce di compiere una breve ricerca su tre intellettuali che, in misura diversa e con diversi gradi di colpa, si resero complici del Terzo Reich, quali Martin Heidegger, Johannes Stark e Micheal von Faulhaber; nel secondo si chiede di ricostruire la biografia di Albert Speer, "l'architetto di Hitler". Quando possibile, negli esercizi si consiglia di far seguire all'attività di ricerca, individuale o di gruppo, un momento di restituzione condivisa e di discussione in classe, affinché il confronto fra pari consolidi e fissi le conoscenze acquisite e alleni alla discussione.

\section{CONFRONTI E COLLEGAMENTI}

La storia del Novecento non è l'unico ambito a cui approdano le consegne degli esercizi: infatti l'apertura interdisciplinare de I sommersi e i salvati permette di spaziare fra saperi diversi e di stabilire collegamenti per nulla scontati fra ambiti apparentemente lontani fra loro. Un gruppo di esercizi disseminati nei sette capitoli dell'opera mette in contatto I sommersi e i salvati con testi di altri autori o con opere diverse dello stesso Levi; gli accostamenti interessano generi ed epoche differenti: ad esempio la riflessione sull'uomo come animale gregario nel capitolo La zona grigia offre il pretesto per un confronto con le nozioni di animale sociale, gregario e monadico contenute nella Politica di Aristotele[4], mentre in un esercizio riferito al capitolo Stereotipi il fulcro dell'attenzione si sposta sul rapporto fra reduci e racconto del passato attraverso l'analisi delle figure di Ulisse nell'Odissea e di Francesca da Rimini nella Divina commedia.

\section{LA RIFLESSIONE SUL PRESENTE}


Nello stesso capitolo è presente una proposta di attività in classe il cui obiettivo è quello di avvicinare le questioni dibattute nel libro al presente degli studenti. Si richiede infatti di compilare un elenco di domande che si vorrebbero rivolgere a Levi oggi e poi di discuterne in classe. In seguito i ragazzi sono invitati a leggere l'Appendice all'edizione scolastica di Se questo è un uomo del 1976e a confrontare le domande formulate da loro e quelle poste dagli studenti degli anni Sessanta e Settanta. L'esame comparato delle due liste di domande consente di mettere a fuoco i tratti salienti della diversa ricezione degli stessi contenuti da parte di generazioni di lettori differenti e al tempo stesso di studiare, per mezzo di un esempio concreto, l'evoluzione delle forme culturali che determinano sia le modalità di lettura e di rielaborazione individuale sia l'approccio didattico ai testi, trasmesso dai docenti.

\section{IL FASCICOLO PER L'INSEGNANTE}

Al volume annotato de I sommersi e i salvati si accompagna un fascicolo omaggio riservato agli insegnanti. Il fascicolo si compone di tre sezioni: 1) una serie di percorsi di apprendimento cooperativo, cinque in tutto, su alcuni temi chiave del libro; 2) undici percorsi di analisi guidata di testi di Primo Levi, sul modello delle prove di tipologia A e B dell'esame di stato; 3) una ricognizione bibliografica e sitografica sulla figura di Primo Levi, sulla storia della Resistenza e della deportazione e sulla didattica della Shoah.

\section{L'APPRENDIMENTO COOPERATIVO}

Nella prima sezione sono proposti cinque percorsi di apprendimento cooperativo aventi ciascuno un tema che prende spunto da un capitolo de I sommersi e i salvati: tre sono incentrati sulla memoria, in tre differenti accezioni (memoria biologica, memoria collettiva, metafore della memoria); uno è dedicato alla zona grigia; l'altro allo stereotipo del prigioniero. Si è pensato a un lavoro a gruppi improntato su ricerca, azione e restituzione, da svolgersi direttamente sui testi, sulle immagini, sui video. I testi scelti spaziano dalla tradizione letteraria (Montale, Borges, Shakespeare, Dostoevskij), filosofica (Platone, Cicerone, Agostino, Bergson, Freud, Arendt), scientifica (Alexander Lurija) e storico-memorialistica (Massimo Mila, Luciana Nissim) con incursioni nel fumetto (Maus di Art Spiegelman, ma anche le vignette di prigionia di Ernesto Rossi) e nell'arte figurativa (il memoriale di Berlino, le Stolpensteine). Si propone anche la visione e la discussione di film e serie tv (Prison Break, Black Mirror[5]) per riflettere sull'uso, stereotipato o originale, di alcuni dei temi scelti. I cinque percorsi si propongono come altrettanti itinerari interpretativi, costruiti però dagli studenti attraverso un rapporto attivo con il materiale testuale e audiovisivo. Ciascun percorso è scandito in tre fasi: nella prima, la classe fruisce di un testo o di un video collettivamente; nella seconda fase, gli studenti sono divisi in gruppi, e ad ognuno è affidato un testo o 
un'immagine (o serie di immagini) da analizzare e approfondire. Nella fase finale, ciascun gruppo restituisce alla classe il proprio approfondimento, per innescare una discussione complessiva sul tema.

L'obiettivo è quello di far reagire alcuni brani di Levi - tratti dai Sommersi, ma anche da altre sue opere meno battute - con alcuni dei testi e degli autori più importanti della tradizione, ma anche di ibridarli con nuovi linguaggi contemporanei.

\section{CAPITOLI, I TEMI, I CONFRONTI}

Il percorso dedicato alla zona grigia, ad esempio, assegna a sei gruppi di studenti sei temi presenti nell'omonimo capitolo de I sommersi e i salvati (l'isolamento e la mancanza di solidarietà, il contagio del male, la vicenda di Chaim Rumkowski, il decano del ghetto di Łódź, il giudizio morale sulla condotta dell'individuo, il male burocratico, il potere vicario) e li sviluppa arricchendo il punto di vista su di essi attraverso il confronto con voci provenienti dalla filosofia, dalla storia, dalla tradizione letteraria, dal fumetto. Gli studenti che si occupano della riflessione sull'isolamento e la mancanza di solidarietà approfondiscono un brano de Le origini del totalitarismo[6] di Hannah Arendt; il gruppo che affronta il discorso sul privilegio in Lager basa il suo ragionamento sugli input provenienti da una vignetta tratta da Maus[7] di Art Spiegelman e da un brano di un'intervista a Vladek Spiegelman, padre di Art; un estratto dell'intervista fatta dal regista Claude Lanzman a Benjamin Murmelstein[8], ultimo Decano del Consiglio degli Ebrei di Theresienstad, aiuta il gruppo che si occupa della figura di Chaim Rumkowski a osservare la storia del decano di Łódź da un’angolazione diversa rispetto a quella adottata da Levi; per indagare il problema del giudizio morale sulla condotta dell'individuo alcuni studenti si confrontano con la favola della vecchia e della cipollina tratta da Ifratelli Karamazov[9] di Fedor Dostoevskij, a cui Levi stesso fa riferimento nel suo libro; un brano de La banalità del male[10] di Hannah Arendt pone i ragazzi faccia a faccia con il concetto del male "burocratico", quello perpetrato dai funzionari pronti a mettere la loro firma in calce a qualsiasi provvedimento emanato dallo Stato nazista; la forza di corruzione del potere, fenomeno presente in tutte le collettività umane, è esemplificata da alcuni versi dell'opera Misura per misura di William Shakespeare citati dallo stesso Levi.

Un altro esempio utile per comprendere lo sforzo di collocare il testo leviano al crocevia di linguaggi e discipline diverse è offerto dal percorso di apprendimento cooperativo sulla figura del prigioniero, che forse più di altri si fa portatore manifesto dell'intento ambizioso che percorre l'intera edizione scolastica: gli studenti, opportunamente guidati, possono imparare attraverso la lettura delle opere di Levi a interpretare e a interrogare il presente e non soltanto il passato. Il percorso si ricollega ai contenuti del capitolo Stereotipi, in cui l'autore riflette sul fatto che, con il passare del tempo, i giovani che incontra nelle scuole non riescono più a immaginare le condizioni fisiche e psicologiche dei deportati, l'annientamento del 
corpo che andava di pari passo con quello del pensiero; a questa immagine di tragica impotenza, con la quale le generazioni nate negli anni Sessanta e Settanta non sanno più rapportarsi, si contrappone il mito del prigioniero che "spezza le catene", diffuso dalla letteratura e dal cinema. Per il percorso di apprendimento cooperativo è stato affiancato a un testo tratto da Oro - (Il sistema periodico[11]) in cui Levi racconta la sua prigionia in Valle d'Aosta - un brano tratto da Ricordi della casa dei morti[12] di Luciana Nissim Momigliano sulla "morte interiore" dei prigionieri; insieme ad essi, è proposto un estratto da Le loro prigioni[13] di Massimo Mila, in cui l'intellettuale antifascista racconta - con una certa disincantata ironia il periodo di prigionia a Regina Coeli, la difficoltà nello scrivere lettere che eludessero la censura, la noia, la lettura, le conversazioni, l'avidità di notizie sul presente. Il testo è accompagnato da una vignetta di Ernesto Rossi, che con Mila condivideva la cella in quegli anni. A questi testi si aggiunge la proposta di analizzare e commentare un film che Levi cita nel capitolo, Io sono un evaso[14] (1932), accostandolo a un altro film sullo stesso filone, uscito negli anni settanta, Fuga da Alcatraz[15] (1979), e a una serie tv recente, Prison break[16] (2005-2017). In modo diverso tutti e tre alimentano lo stereotipo del prigioniero forte e padrone di sé, che riesce a liberarsi e a fuggire, spezzando i propri vincoli. La scelta di autori come Nissim e Mila, così come della vignetta di Rossi, ha come obiettivo quello di far incontrare gli studenti con autori che probabilmente non conoscono; la proposta di Prison break, serie tv piuttosto conosciuta dagli adolescenti nati negli anni Duemila, è dettata invece dall'esigenza di creare un ponte fra contenuti eterogenei per abituare gli studenti a riconoscere non solo gli stereotipi storici, ma anche le costruzioni simboliche e i miti letterari che popolano l'immaginario contemporaneo, e a compiere un esercizio di analisi e di lettura del presente.

\section{PERCORSI DI ANALISI TESTUALE}

I brani scelti per i percorsi di analisi del testo sono tratti da Il sistema periodico (racconti Zinco, Potassio, Nichel, Fosforo, Oro, Vanadio), da La chiave a stella, da Racconti e saggi, dalla raccolta di poesie Ad ora incerta, dall'Appendice a Se questo è un uomo. Si è voluto infatti presentare agli studenti un ritratto a tutto tondo dello scrittore, che valorizzasse le molteplici sfaccettature della sua opera e mettesse in discussione la vulgata che considera Primo Levi esclusivamente un testimone - anzi, il testimone per eccellenza - passando sotto silenzio la straordinaria ricchezza di un percorso umano e intellettuale ramificato in molte direzioni e, proprio per questo, fuori dagli schemi.

\section{TESTI E LA PROSPETTIVA INTERDISCIPLINARE}

La maggior parte dei testi tratti da Il sistema periodico ci mostra un Levi poco più che ventenne alle prese con le amicizie, con lo studio della chimica, con le prime esperienze lavorative, con le scelte che segnarono il suo destino e quello della sua generazione, sullo sfondo dell'Italia del fascismo e della Seconda Guerra 
Mondiale. Per il pubblico scolastico questo è, per certi versi, un Primo Levi inedito con il quale è possibile stabilire un rapporto di maggiore vicinanza e forse anche di identificazione.

Se si prova a passare brevemente in rassegna alcuni esempi fra quelli contenuti nella sezione, ci si accorge che anche nei percorsi è adottata una prospettiva interdisciplinare che fonde letteratura, storia del Novecento e storia del costume, studio del pensiero scientifico e riflessione sull'attualità. La sezione si apre con un brano del racconto Zinco in cui Levi, studente di Chimica nell'anno accademico 1938-39, dall'osservazione del comportamento della materia che ha tra le mani in laboratorio, trae alcune considerazioni sui concetti di "purezza" e di "impurezza" ricollegandoli alla propaganda antisemita dell'Italia fascista. Dopo aver svolto un'analisi guidata del brano, lo studente è invitato a sviluppare i concetti di "impurezza" e "diversità" mettendoli in relazione con le conoscenze scientifiche attuali. Due analisi testuali hanno come fulcro il tema del lavoro, declinato da due punti di vista differenti: la riflessione sui sentimenti individuali che legano l'uomo al suo lavoro quotidiano, sollecitata dalla lettura del brano di Nichel che racconta del primo vero impiego di chimico trovato da Levi; il confronto storico fra gli antichi mestieri artigianali, assai diffusi fino alla metà del secolo scorso, e il lavoro nella società contemporanea, condotto a partire dalla figura del padre di Tino Faussone, protagonista del capitolo "Battere la lastra" de La chiave a stella.

Un altro percorso propone l'analisi della poesia Partigia, nella quale lo scrittore nel tracciare un bilancio dell'esperienza resistenziale sua e della sua generazione allarga il discorso dalla sfera storico-politica a quella esistenziale e invita quanti hanno combattuto la lotta contro il nazifascismo a tener saldi i valori che li hanno ispirati in quella stagione ormai lontana.

I due percorsi di analisi sul modello della prova d'esame di tipologia B (analisi e produzione di un testo argomentativo) hanno come oggetto due brani dell'Appendice a Se questo è un uomo. I temi portati all'attenzione degli studenti sono le radici storiche dell'antisemitismo tedesco e i motivi per cui l'opinione pubblica tedesca non "volle sapere" quanto stava accadendo nei campi di prigionia e di sterminio.

\section{BIBLIOGRAFIA E SITOGRAFIA SULLA FIGURA DI PRIMO LEVI, SULLA STORIA DELLA RESISTENZA E DELLA DEPORTAZIONE E SULLA DIDATTICA DELLA SHOAH}

Nella parte dedicata a Levi si è preferito suggerire, anziché contributi critici in senso stretto, testi che offrissero un punto di vista originale sulla biografia intellettuale dell'autore e che potessero essere proposti agli studenti, come ad esempio il graphic novel Una stella tranquilla: ritratto sentimentale di Primo Levi[17], di Pietro Scarnera, e l'Album Primo Levi[18], curato dal Centro Studi Primo Levi. Oltre ai testi, sono descritti siti nei quali si trovano materiali e approfondimenti su Primo Levi, come il "Laboratorio Levi”[19] (su Raiplay), una serie di interviste rilasciate dallo scrittore ai microfoni della Rai. La sezione sulla storia della Resistenza e della deportazione elenca e descrive le risorse on line messe a disposizione dagli istituti, dagli enti e dalle associazioni, italiani e internazionali, che si occupano di questi due temi e, più in generale, di 
storia del Novecento. L'ultima parte della bibliografia ricostruisce per grandi linee il dibattito sulla didattica della Shoah che si è sviluppato a partire dagli anni Novanta del Novecento. Mentre nelle altre due sezioni i contributi citati sono disposti in ordine alfabetico, nell'ultima parte si è optato per un elenco in ordine cronologico per permettere ai docenti di orientarsi fra le diverse fasi del dibattito. Una riflessione preliminare sulla didattica della Shoah da parte dei docenti di Lettere e di Storia appare oggi più che mai necessaria: la bibliografia vorrebbe quindi facilitare l'acquisizione di una formazione specifica in materia, la quale va di pari passo con un incremento della consapevolezza delle implicazioni educative, cognitive e metodologiche connesse all'adozione di un determinato approccio didattico. Avere l'opportunità di vagliare l'ampia gamma di posizioni assunte dagli studiosi e le tante proposte di attività didattiche sul tema significa già, per il docente, iniziare a compiere quelle scelte che sente più congeniali rispetto alla sua sensibilità, alle sue competenze e agli obiettivi formativi, al fine di trasmettere non un semplice insieme di nozioni, bensì un bagaglio di conoscenze complesse.

\section{Note:}

[1] Primo Levi, Se questo è un uomo, Torino, Einaudi 1973, collana "Letture per la scuola media".

[2] Victor Klemperer, LTI. La lingua del Terzo Reich. Taccuino di un filologo [1946], Firenze, Giuntina, 1998.

[3] Aleksandr Solzenicyn, Arcipelago Gulag, 1918-1956: saggio di inchiesta narrativa [1973], Milano, Mondadori, 2013.

[4] Cfr., in particolare, Aristotele, Historia Animalium, I, 488a e Politica, I, 1253a.

[5] Black Mirror, serie TV, di Charlie Brooker, Regno Unito, 2011- in produzione, cinque stagioni.

[6] Hannah Arendt, Le origini del totalitarismo [1951], Torino, Einaudi, 2004.

[7] Art Spiegelman, Maus [1972-1991], Torino, Einaudi, 2010.

[8] Claude Lanzmann, L'ultimo degli ingiusti. Intervista con Benjamin Murmelstein, ultimo Decano del Consiglio degli Ebrei di Theresienstadt, Milano, Skira, 2014

[9] Fedor Dostoevskij, I fratelli Karamazov [1878-1880], a cura di Igor Sibaldi, voll. I-II, Milano, Mondadori, 1994.

[10] Hannah Arendt, La banalità del male: Eichmann a Gerusalemme [1963], Milano, Feltrinelli, 2008.

[11] Per tutte le opere di Primo Levi citate si rimanda alle Opere complete, a cura di Marco Belpoliti, voll. IIII, Torino, Einaudi, 2016-2018.

[12] Luciana Nissim Momigliano, Ricordi della casa dei morti [1946] in Ricordi della casa dei morti e altri scritti, a cura di Alessandra Chiappano, Firenze, Giuntina, 2008.

[13] Massimo Mila, Le loro prigioni. Da Regina Coeli a Ventotene, «Il Ponte», V, 3, marzo 1949; ora in Scritti civili, a cura di Alberto Cavaglion, Milano, Il Saggiatore, 2011. 
[14] Io sono un evaso, film, regia di Mervyn Le Roy, USA 1932.

[15] Fuga da Alcatraz, film, regia di Don Siegel, USA 1979.

[16] Prison Break, serie TV, di Paul Scheuring, USA 2005-2017,cinque stagioni.

[17] Pietro Scarnera, Una stella tranquilla: ritratto sentimentale di Primo Levi, Bologna, Comma22, 2013.

[18] Album Primo Levi, a cura di Roberta Mori e Domenico Scarpa, Torino, Einaudi, 2017.

[19] Cfr. https://www.raiplay.it/programmi/laboratoriolevi/ (URL consultato il 18/06/2019) 